\title{
Insolation Forecasting Method Using NN with EPSO in Consideration of Correlation between Other Regions
}

\author{
Shoji Kawasaki and Toshiya Hoga \\ Department of Electronics and Bioinformatics, School of Science and Technology, Meiji University, Kanagawa, 2148571, Japan
}

\begin{abstract}
Recently, there is a movement that aims to realize a distributed energy system in Japan. The PV (photovoltaic) generation is especially expected, and it is anticipated that interconnection number of PVs will increase more and more in the future. However, an amount of insolation is easily affected by the weather, the output of PV and the supply of electricity becomes unstable. And it causes various problems in a distribution system. Therefore, it is important to forecast the amount of insolation. In previous study, MLPNN (multilayer perceptron neural network) is a general method to forecast the amount of insolation. However, it is in danger of falling into a local solution by only MLPNN. In this study, the authors propose a forecasting method of amount of insolation using MLPNN and EPSO (evolutionary particle swarm optimization). The authors use EPSO in addition to MLPNN to solve the problem. The authors also propose a forecasting method of amount of insolation using other regions weather data for the accuracy improvement.
\end{abstract}

Key words: PV generation, amount of insolation, neural network, EPSO.

\section{Introduction}

In recent years, there is a movement that aims to realize a distributed energy system from the standpoints of taking measures against the environmental issues and reducing dependency on nuclear power in Japan. The PV (photovoltaic) generation is especially expected, and it is anticipated that interconnection number will increase more and more in the future. However, the output of PV is unstable and varies greatly due to amount of insolation, temperature of panel and weather conditions. Therefore, there is concern that various power quality deteriorates. For example, there is a possibility that deviates from the voltage proper range, since the output of PV cannot maintain a stable power supply. Hence, the studies on the mitigation of output fluctuation of PV by the installation of electric storage device $[1,2]$, or the studies on the control for the voltage deviation of electric power system have been actively carried out $[3$, 4]. It may be possible to control the voltage of power

Corresponding author: Shoji Kawasaki, Eng. Dr., associate professor, research fields: advancement of electric power system, improvement of electric power quality. system efficiently than ever by incorporating a forecast of PV output into the voltage problems. Moreover, it is possible to develop the optimum operation plan of the power-generation facilities than ever. Not only that, the surplus power can be relaxed, and shortage of electric power supply can be eliminated.

For the reasons described above, the studies on the forecast of PV output are actively investigated [5-7]. However, when forecasting output of PV, the forecasting results will be specialized in installation environment and characteristics of the panel. Forecasting amount of insolation is more versatile than forecasting output of PV, since it can be calculated by the conversion formula. Therefore, in this study, the authors forecast amount of insolation.

The biggest goal of this study is to develop a practical forecasting method of amount of insolation with high precision. In this study, the authors propose a forecasting method of amount of insolation by using the MLPNN (multilayer perceptron neural network) and the EPSO (evolutionary particle swarm optimization) based on the past measured data which Japan Meteorological Agency has published. EPSO has the advantage of obtaining better solution by 
applying evolutionary strategy to PSO [8]. Various optimization methods such as NN [5], genetic algorithm [9], and so on are used for the forecasting of amount of insolation or output of PV. Also, in the previous studies, there is the forecasting method of output of PV by the generalized radial basis function network (GRBFN) with EPSO [6], and the method using MLPNN with PSO. However, the forecast of amount of insolation using MLPNN with EPSO throughout the year has not been carried out. From the above, the authors propose a forecasting method of amount of insolation throughout the year by MLPNN with EPSO, and verify the usefulness of proposed method. In addition, the authors also propose the correction of forecasting accuracy that takes into account the correlation of weather data between other regions and forecasting point.

\section{Proposed Forecasting Method}

\subsection{Configuration of $N N$}

$\mathrm{NN}$ is an optimization method simulating neural circuit of human brain. There are several kinds of NN, such as feed forward NN and recurrent NN. In this study, the authors use MLPNN. The MLPNN of this study consists of three layers: input layer, hidden (intermediate) layer, and output layer. A unit exists in each layer. There are weights between each layer, and thresholds exist in each unit. These values are updated by back propagation. It is a method of updating weights and thresholds based on the error between output obtained by forward propagation and supervised data. Fig. 1 shows a conceptual diagram of MLPNN. In Fig. $1, y$ : input data, $i$ : number of unit in input layer, $j$ : number of unit in hidden layer, $k$ : number of unit in output layer, $I$ : output at input layer, $H$ : output at hidden layer, $O$ : output at output layer, $z$ : output data, $W$ : weight between input layer and hidden layer or hidden layer andoutput layer.

\subsection{Configuration of EPSO}

This section explains EPSO that adds evolutionary strategy with PSO. EPSO has been proposed in previous study [8]. PSO is one of the metaheuristic methods that simulates the behavior of swarm of birds or fish evolving while sharing the individual information making up the group. And it is known as one of the effective methods for solving optimization problem. The best solution in each search process is held in each individual. Furthermore, the best solution of whole swarm (global best) is obtained by sharing each best solution (personal best).

The movement rules of each individual are expressed by Eqs. (1) and (2). The conceptual diagram of PSO is shown in Fig. 2. In Fig. 2, pbest: personal best, gbest: global best.

$$
\begin{gathered}
v_{d}^{c+1}=w_{1} * v_{d}^{c}+w_{2} * \text { rand } *\left(\text { pbest }_{d}-x_{d}^{c}\right) \\
+w_{3} * \text { rand } *\left(\text { gbest }-x_{d}^{c}\right) \\
x_{d}^{c+1}=x_{d}^{c}+v_{d}^{c+1}
\end{gathered}
$$

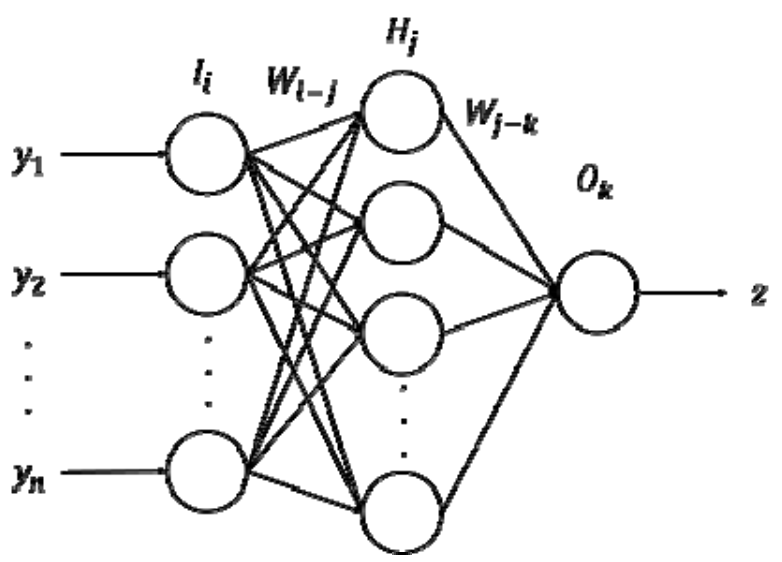

Fig. 1 Conceptual diagram of NN.

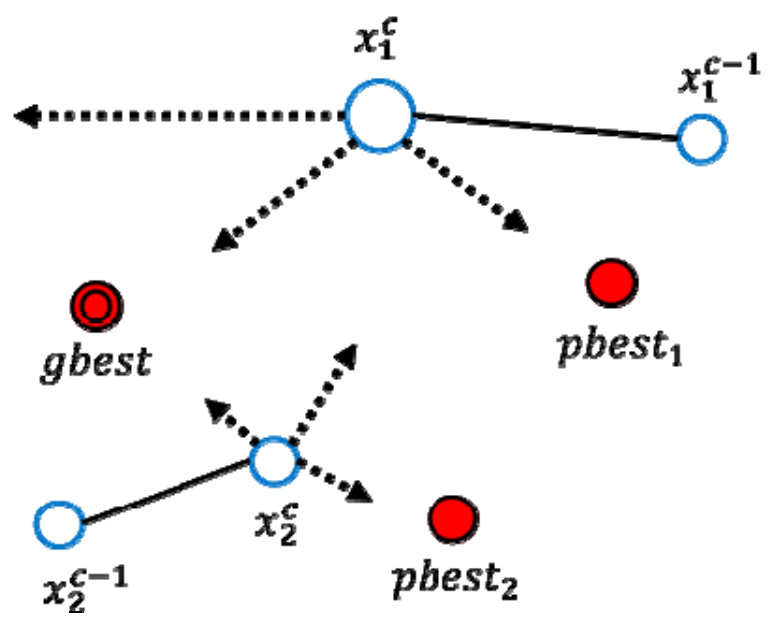

Fig. 2 Conceptual diagram of PSO. 
where, $v$ : speed, $x$ : position, $c$ : current search count, $d$ : individual number, $w_{1} \sim w_{3}$ : weight, rand: random number.

However, when one individual reaches a local search solution, other individuals may be affected by it and become invariant. Therefore, in order to solve this problem, EPSO that adds an evolutionary strategy to PSO has been proposed [8]. By considering evolutionary strategy of duplication and mutation in each individual, randomness is given to them and breaks away from the local search solution.

The movement and mutation rules of each individual are shown below.

$$
\begin{gathered}
v_{d}^{c+1}=w_{1}^{\prime} * v_{d}^{c}+w_{2}^{\prime} * \text { rand } *\left(\text { pbest }_{d}-x_{d}^{c}\right) \\
+w_{3}^{\prime} * \text { rand } *\left(\text { gbest }^{\prime}-x_{d}^{c}\right) \\
w_{a}^{\prime}=w_{a}+\tau_{1} N(0,1) \\
\text { gbest } t^{\prime}=\text { gbest }+\tau_{2} N(0,1)
\end{gathered}
$$

where, ': mutation, $a: 1-3, \tau_{1}, \tau_{2}$ : learning parameters (constant), $N(0,1)$ : a random number following the normalized Gaussian distribution with 0 mean and variance 1 .

The procedure of EPSO is shown below.

[Step 1] Determination of each parameter and maximum number of searches.

[Step 2] Consideration of evolutionary strategy.

Replication: Replicate each individual.

Mutation: Mutations apply to offspring individual.

Movement: Move each individual based on movement rules.

Selection: Select the parent or offspring individual in each individual according to an arbitrary selection rule. In this study, the authors used tournament selection.

Evaluation: Evaluate each individual.

[Step 3] Find the best solution of personal and group.

[Step 4] When the upper limit of the number of searches has been reached, the process is terminated. Otherwise, return to [Step 2].

\subsection{Configuration of NN with EPSO}

This section explains the proposed method. In this study, EPSO is applied to MLPNN mentioned above. In the proposed method, $i$ units (at input layer), $j$ units (at hidden layer), and $k$ units (at output layer) are prepared. Therefore, there are $(i+j+k)$ thresholds. Also, there are $\{(i * j)+(j * k)\}$ weights. So, the total number of search points covered by one individual in PSO or EPSO is $\{(i+j+k)+(i * j)+$ $(j * k)\}$ points.

The flowchart of the proposed learning algorithm is shown in Fig. 3. First, each parameter is defined. And, the weather data are applied to MLPNN. Next, each weight and threshold of MLPNN is converted to individuals in EPSO, and the optimum solution is updated using the supervised data, and those values are evaluated. A better weight and thresholds can be obtained by performing above procedure while defining number of searches.

\section{Example of Forecasting Results}

\subsection{Conditions for Forecasting}

In this study, the authors use past measurement data

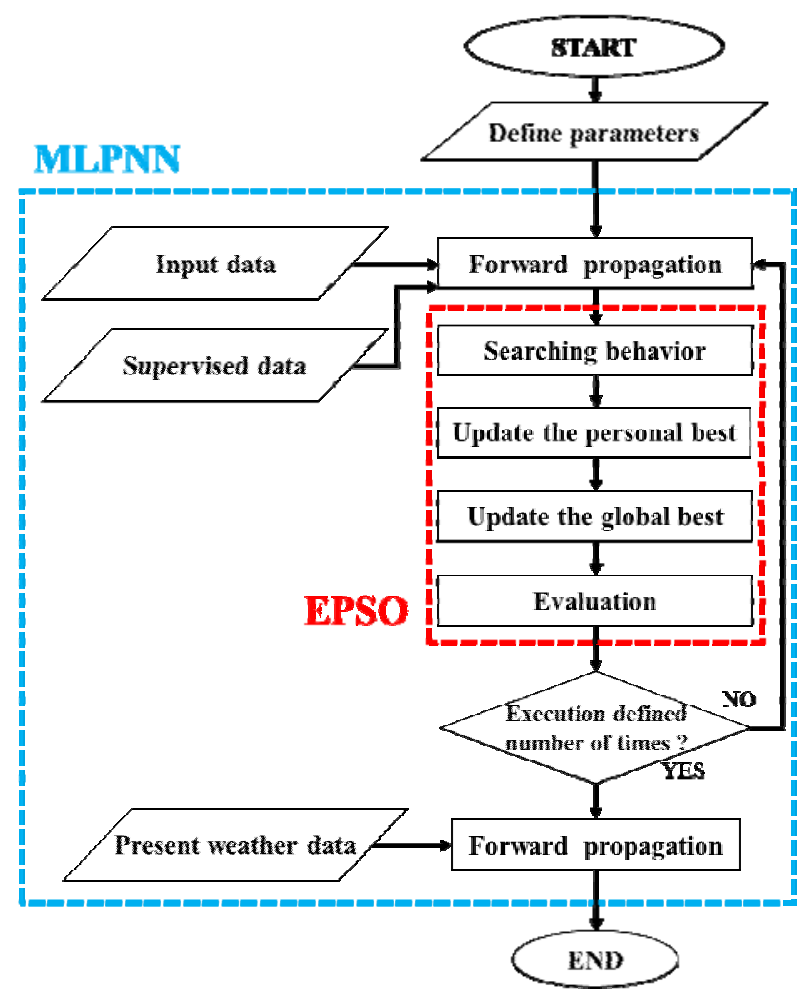

Fig. 3 Flowchart of proposed algorithm. 
Table 1 Parameter of PSO and EPSO.

\begin{tabular}{lll}
\hline & PSO & EPSO \\
\hline$w_{1}$ & 0.7 & 0.7 \\
$w_{2}$ & 1.5 & 1.5 \\
$w_{3}$ & 1.5 & 1.5 \\
$\tau_{1}$ & - & 0.05 \\
$\tau_{2}$ & - & 0.05 \\
$d$ & 50 & 50 \\
$c$ & 300 & 300 \\
\hline
\end{tabular}

as forecasting data, since there are no accumulated forecasting data. The types of used data are shown below. As input data, the authors use five elements (time, atmospheric pressure, temperature, humidity, and wind speed), and one element of insolation as supervised data. The reason for using these data is that these are observed in most areas. These weather data are measured data of Tokyo released by Japan Meteorological Agency [10]. Both input data and supervised data are normalized to $0 \sim 1$ for calculation.

The learning period is three years from January 1, 2013 to December 31, 2015. In addition, the forecasting period is one year from January 1, 2016 to December 31, 2016. The learning time is divided into wintertime (Jan., Feb., Dec.), summertime (Jun., Jul., Aug.), and other time (Mar., Apr., May, Sep., Oct., Nov.), and the learning algorithms are constructed respectively in each time period. Missing data are excluded.

The parameters of PSO and EPSO are shown in Table 1. The authors forecasted amount of insolation using the following three methods for comparison.

[Method 1] Forecasting method using MLPNN

[Method 2] Forecasting method using MLPNN with
PSO (MLPNN + PSO)

[Method 3] Forecasting method using MLPNN with EPSO (MLPNN + EPSO)

\subsection{Forecasting Results}

In order to verify the validity of the proposal method, the accuracy verification throughout the year (from Jan. 1, 2016 to Dec. 31, 2016) was carried out. The MAE (mean absolute error) and RMSE (root mean squared error) were used for the evaluation (see Eqs. (6) and (7)). The evaluation was carried out at the time zones with sunshine (from 7:00 to 17:00) throughout the year.

$$
\begin{aligned}
& M A E=\frac{1}{n} \sum_{d=1}^{n}\left|I_{d t}-I^{*}{ }_{d t}\right| \\
& R M S E=\sqrt{\frac{1}{n} \sum_{d=1}^{n}\left(I_{d t}-I^{*}{ }_{d t}\right)^{2}}
\end{aligned}
$$

where, $n$ : number of data, $d$ : date, $t$ : time, $I_{d t}$ : amount of observed insolation, $I^{*}{ }_{d t}$ : amount of forecasting insolation.

Fig. 4 shows an example of the forecasting results and observed values from Aug. 1 to 10 in 2016. The annual forecasting accuracy is shown in Fig. 5 and Table 2. As shown in Fig. 5, the forecasting accuracy was improved by adding PSO or EPSO to MLPNN. In case the method by only MLPNN, the forecasting accuracy got worse in summertime. On the other hand, in case the method by MLPNN with PSO or EPSO, the accuracy in summertime improved to the same accuracy with wintertime. As shown in Table 2, the forecasting accuracy was improved throughout the year in case the method by MLPNN with PSO or EPSO.

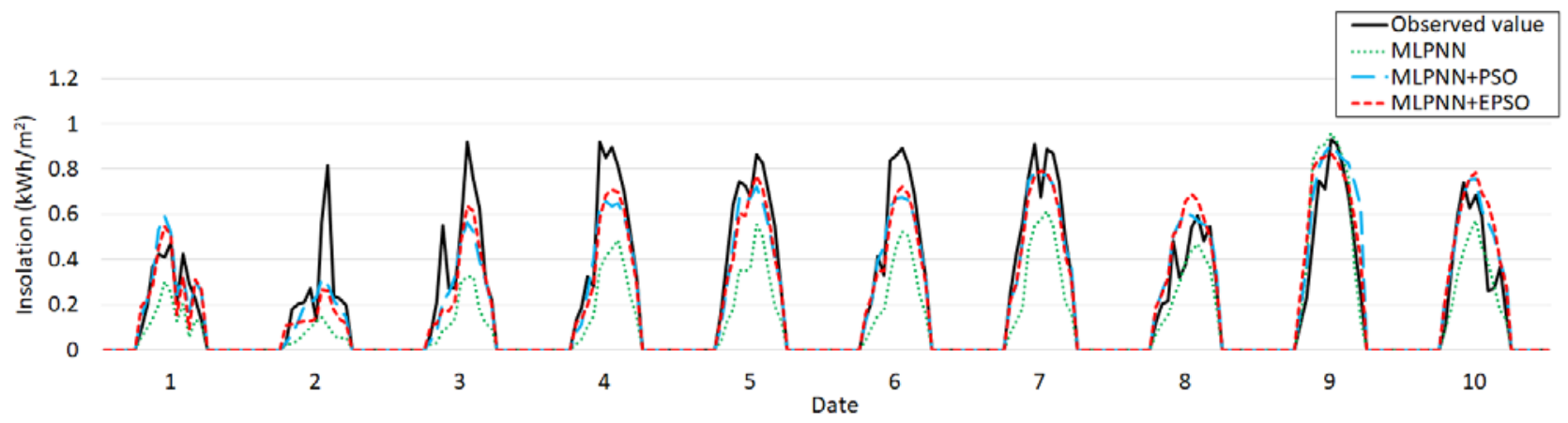

Fig. 4 Results of insolation forecasting (Tokyo, Aug. 1-10, 2016). 


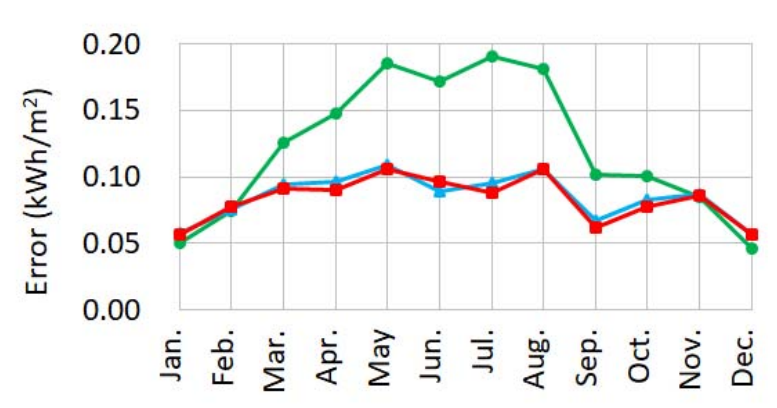

(a) MAE

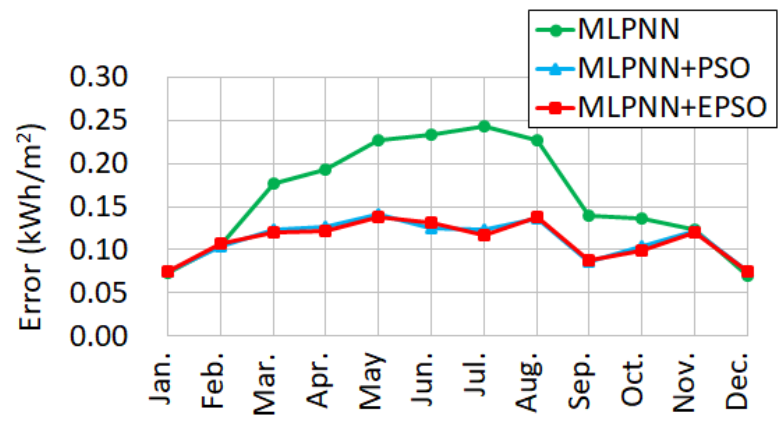

(b) RMSE

Fig. 5 Results of monthly accuracy verification (Tokyo, 2016).

Table 2 Results of annual accuracy verification(Tokyo, 2016).

\begin{tabular}{lll}
\hline & $\begin{array}{l}\text { MAE } \\
\left(\mathrm{kWh} / \mathrm{m}^{2}\right)\end{array}$ & $\begin{array}{l}\text { RMSE } \\
\left(\mathrm{kWh} / \mathrm{m}^{2}\right)\end{array}$ \\
\hline MLPNN & 0.122 & 0.162 \\
MLPNN + PSO & 0.085 & 0.112 \\
MLPNN + EPSO & 0.083 & 0.111 \\
\hline
\end{tabular}

Compared to the method by only MLPNN, the annual average accuracy of the method by MLPNN with PSO was improved by $30.3 \%$ (MAE) or $30.9 \%$ (RMSE) and the accuracy of the method by MLPNN with EPSO was improved by $32.0 \%$ (MAE) or $31.5 \%$ (RMSE).

As shown in Table 2, the forecasting accuracy by MLPNN was poor. It is important to improve the forecasting accuracy by MLPNN, since MLPNN is used for all methods ([Method 1]-[Method 3]). In the future, the authors are going to investigate the optimum number of parameters like layers and units. In addition, the authors are going to try to use other NN method.

\section{Forecasting Method Using Weather Data of Other Regions}

\subsection{Investigative Methods of Correlation}

The authors tried to improve the accuracy by adding weather data around forecasting target point. The investigative method of correlation with weather data in other regions is shown below.

[Step 1] Select around regions of forecasting target point.

[Step 2] Investigate the correlation of weather data between forecasting target point and other regions from five hours ago to the forecasting hour.

[Step 3] Investigate the correlation between the amount of insolation at the forecasting target point and the weather data at the correlation investigation point.

[Step 4] Based on the correlation of Steps 2 and 3, select a region with most strong correlation at the same time.

In this study, since the authors made forecasts in Tokyo, the authors selected 10 points (Kumagaya, Maebashi, Kofu, Mito, Utsunomiya, Yokohama, Chiba, Fukushima, Niigata, and Nagano) as other regions (see Fig. 6a). The authors chose these regions since there is large weather observation station. Fig. $6 \mathrm{~b}$ shows the results of Step 1 to Step 4. As shown in Fig. 6b, Kumagaya has the strongest correlation with Tokyo. Therefore, the weather data in Kumagaya are added as input data.

\subsection{Forecasting Results Using Other Region}

The amount of insolation is forecasted using the following three methods.

[Method 4] Forecasting method using MLPNN with other region

(MLPNN + other region)

[Method 5] Forecasting method using MLPNN with PSO and other region

(MLPNN + PSO + other region)

[Method 6] Forecasting method using MLPNN with EPSO and other region

$($ MLPNN + EPSO + other region) 


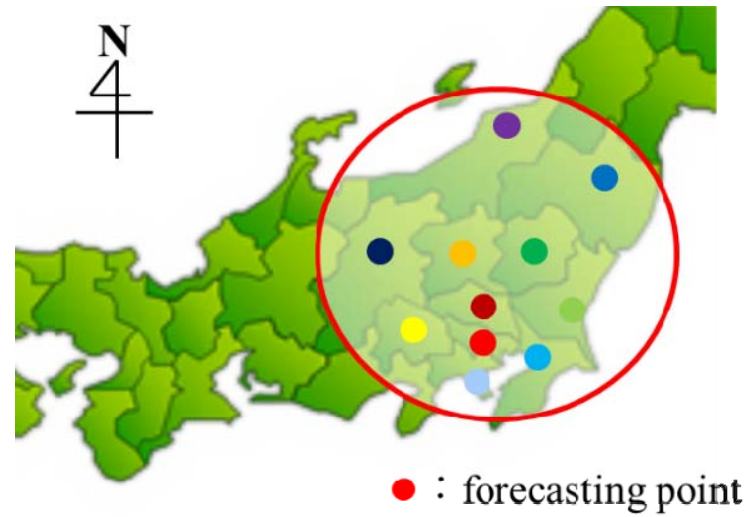

(a) Around regions of forecasted point.

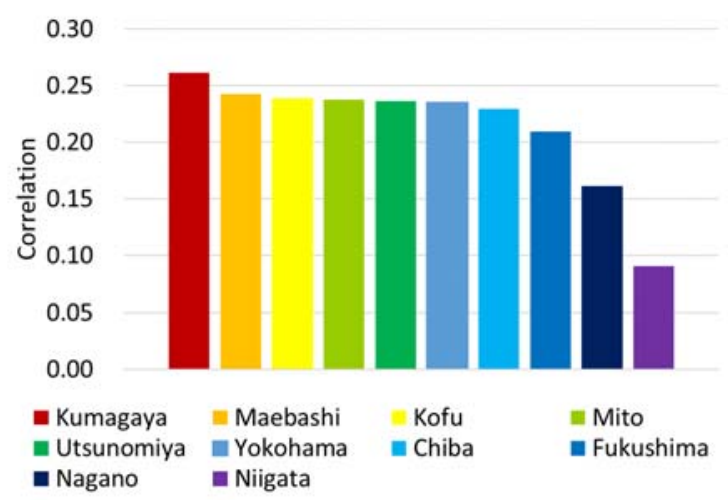

(b) Correlation around regions.

Fig. 6 Investigative results of regions with strong correlation.

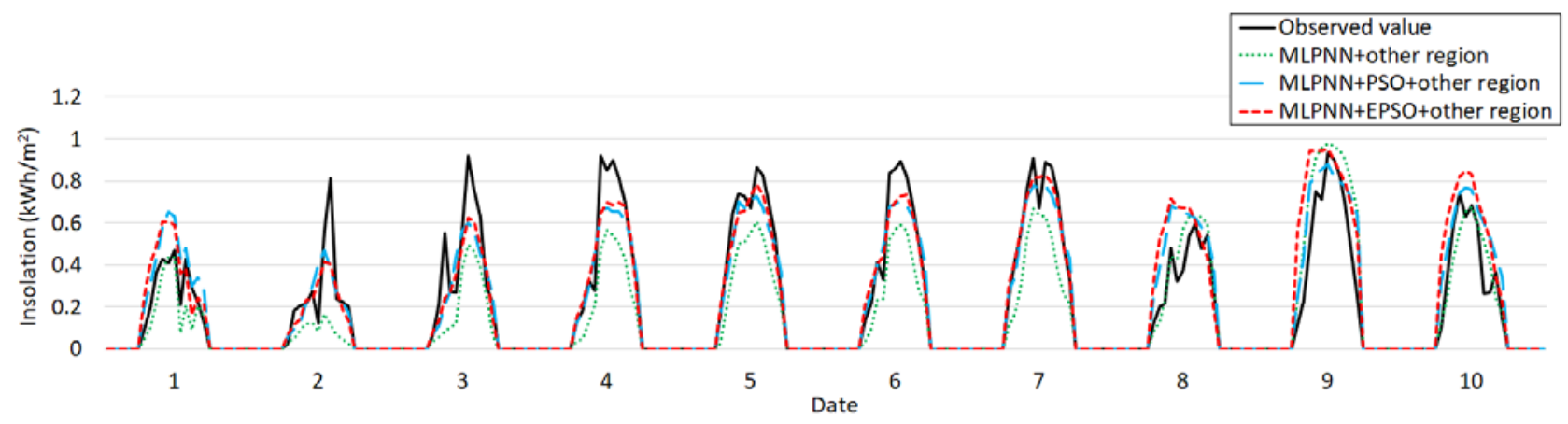

Fig. 7 Results of insolation forecasting by adding data of other region (Tokyo, Aug. 1-10, 2016).

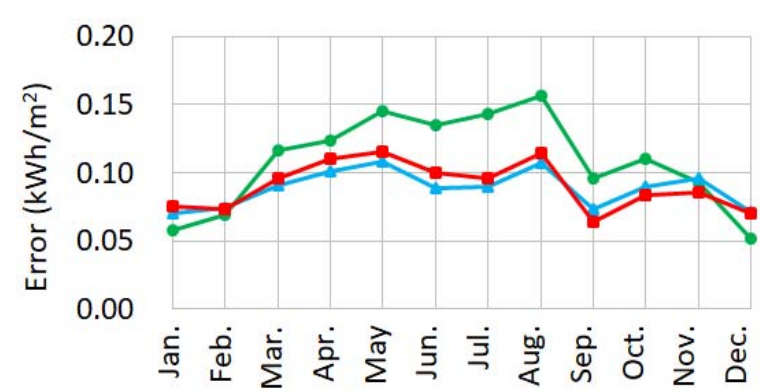

(a) MAE

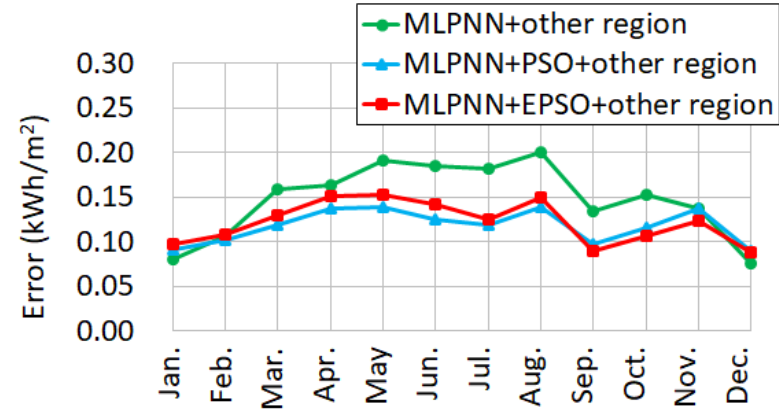

(b) RMSE

Fig. 8 Accuracy verification results using by adding data of other region (Tokyo, 2016).

Table 3 Results of annual accuracy verification by adding data of other region (Tokyo, 2016).

\begin{tabular}{lll}
\hline & $\begin{array}{l}\text { MAE } \\
\left(\mathrm{kWh} / \mathrm{m}^{2}\right)\end{array}$ & $\begin{array}{l}\text { RMSE } \\
\left(\mathrm{kWh} / \mathrm{m}^{2}\right)\end{array}$ \\
\hline $\begin{array}{l}\text { MLPNN } \\
+ \text { other region }\end{array}$ & 0.108 & 0.147 \\
$\begin{array}{l}\text { MLPNN + PSO } \\
+ \text { other region }\end{array}$ & 0.088 & 0.118 \\
$\begin{array}{l}\text { MLPNN + EPSO } \\
+ \text { other region }\end{array}$ & 0.090 & 0.122 \\
\hline
\end{tabular}

Fig. 7 shows an example of forecasting results and observed values from Aug. 1 to 10 in 2016. The annual forecasting accuracy is shown in Fig. 8 and Table 3.

As shown in Fig. 7, the forecasting accuracy was improved by adding PSO or EPSO to MLPNN. From Fig. 8 and Table 3, the accuracy of the method by MLPNN with other region was improved by about 
$11.1 \%$ (MAE) or 9.3\% (RMSE) as compared to the method by only MLPNN. However, in the method by MLPNN with PSO and other region, the accuracy deteriorated as a result of adding weather data in other region. The same is true for the accuracy of the method by MLPNN with EPSO and other region. It turned out that not simply increasing number of input data would improve the accuracy. Therefore, in the future, it should be considered well to the investigative method of correlation with weather data in other regions. In addition, the authors need to consider utilization of other elements (e.g. precipitation, cloud amount) as input data.

\section{Conclusions}

In this study, the authors proposed the forecasting method of amount of insolation applying MLPNN with EPSO. And the authors investigated the forecasting accuracy throughout the year and verified the usefulness of the proposed method.

As a result of the accuracy verification using two kinds of evaluation methods, the accuracy of the method by MLPNN with EPSO was improved by $31.9 \%$ as compared to the method by only MLPNN. From this result, the authors could demonstrate the usefulness of the proposed method. In any method, the forecasted accuracy was inferior in summer compared to winter. It is considered that this is because the amount of insolation is larger in summer than in winter. Therefore, in summer, if forecast is lost, forecasted accuracy also has a big influence. In other words, if we can improve the forecasted accuracy in summer, it will contribute greatly to improving the annual forecasted accuracy.

In addition, the authors tried to improve forecasted accuracy by using weather data of other regions. The authors selected the influential region using our own correlation investigative method. As a result, the forecasted accuracy was improved when the weather data of other region were used for the method by only MLPNN. On the other hand, the forecasted accuracy was deteriorated when the weather data of other region were used for the method by MLPNN with PSO or EPSO.

The future tasks will be described below.

(1) It is necessary to find an appropriate initial value of each parameter of MLPNN, PSO, and EPSO. These values change greatly depending on the initial value to be set. Since these depend on the problem to be solved, it is only decided empirically.

(2) It is necessary to verify the forecasted accuracy in other areas. And the forecasted accuracy should be compared with other methods of previous studies under the same conditions.

(3) It is necessary to examine the correlation investigative method in other regions. In this study, it was effective to use the weather data of other region for the MLPNN method. However, the forecasted accuracy deteriorated with other methods. It is also considered that there was a problem with the correlation investigative method.

(4) The authors will develop an operation plan of the power-generation facilities using the proposed forecasting method. The authors would like to build the optimal operation of power system by applying it to scheduling technology and control system.

\section{References}

[1] Takagi, M., Iwafune, Y., Yamamoto, H., Yamaji, K., Okano, K., Hiwatari, R., and Ikeya, T. 2010. "Countermeasure for Surplus Electricity of PV Using Replacement Battery of EVs." IEEJ Trans. PE 130 (7): 651-60.

[2] Yabe, K., and Yamamoto, H. 2017. "Impact Analysis of Introducing Storage Battery to Districts Variable Renewable Energy Is Largely Installed." IEEJ Trans. PE 137 (1): 16-26

[3] Kondo, T., Baba, J., and Yokoyama, A. 2006. "Voltage Control of Distribution Network with a Large Penetration of Photovoltaic Generations Using FACTS Devices." IEEJ Trans. PE 126 (3): 347-58.

[4] Hayashi, Y. 2009. "Trend and Future View of Voltage Control for Distribution Systems with Distributed Generators." IEEJ Trans. PE 129 (4): 491-4.

[5] Yona, A., Senjyu, T., Yousuf Saber, A., Funabashi, T., Sekine, H., and Kim, C. H. 2008. "Application of Neural Network to 24-Hours-Ahead Generating Power Forecasting for PV System." IEEJ Trans. PE 128 (1): 


\section{Correlation between Other Regions}

34-9.

[6] Takahashi, M., and Mori, H. 2012. "A GRBFN-EPSO-Based Method for Predicting PV Generation Output." IEEJ Trans. PE 133 (1): 72-8.

[7] Sasaki, Y., Yorino, N., Wahyudi, F. I., Seikoba, D., Asada, M., Ma, L., and Zoka, Y. 2017. "A Simple and Reliable PV Forecasting Method for Local Area Energy Management." IEEJ Trans. PE 137 (7): 538-45.

[8] Miranda, V., and Fonseca, N. 2002. "New Evolutionary
Particle Swarm Algorithm (EPSO) Applied to Voltage/Var Control." In Proceedings of PSCC'02-Power System Computation Conference, Sevilla, Spain, June 24-28.

[9] Kawasaki, S., Taoka, H., Nagao, T., and Onaka, K. 2014. "Development of Insolation Forecasting Method by Genetic Algorithm." IEEJ Trans. PE 135 (2): 89-96.

[10] The Homepage of Japan Meteorological Agency. http://www.jma.go.jp/jma/index.html. 